\title{
Three-dimensional Si / vertically oriented graphene nanowalls composite for supercapacitor applications
}

\author{
Shahzad Hussain a,b,c, , Roger Amade ${ }^{\mathrm{a}, \mathrm{b}}$, Adrian Boyd ${ }^{\mathrm{c}}$, Arevik Musheghyan-Avetisyan ${ }^{\mathrm{a}, \mathrm{b}}$, \\ Islam Alshaikh $^{\mathrm{a}, \mathrm{b}}$, Joan Martí-Gonzalez ${ }^{\mathrm{a}, \mathrm{b}}$, Esther Pascual ${ }^{\mathrm{a}, \mathrm{b}}$, Brian J. Meenan ${ }^{\mathrm{c}}$, \\ Enric Bertran-Serra ${ }^{a, b}$ \\ ${ }^{a}$ Department of Applied Physics, Universitat de Barcelona, C/Martí $i$ Franquès, l, 08028, Barcelona, Catalunya, Spain \\ ${ }^{\mathrm{b}}$ ENPHOCAMAT (FEMAN) Group, Institute of Nanoscience and Nanotechnology (IN2UB), Universitat de Barcelona, C/Martí $i$ Franquès, l, 08028, Barcelona, \\ Catalunya, Spain \\ ${ }^{\mathrm{c}}$ Nanotechnology \& Integrated Bio-Engineering Centre (NIBEC), Ulster University, Shore Road, Newtownabbey, BT37 OQB, United Kingdom
}

\section{A R T I C L E I N F O}

\section{Keywords:}

Flexible substrate

Vertically oriented graphene

Nanocomposite

Supercapacitors

\begin{abstract}
A B S T R A C T
Three-dimensional (3D) carbon nanostructures are promising architectures to improve both specific capacity and power density of electrochemical energy storage systems. Their open structure and porosity provide a large space for active sites and high ion diffusion rates. To further increase their specific capacity, they can be combined with metal oxides. However, this combination often results in the loss of cycling stability and power density. Among the different electrode materials being studied, vertically oriented graphene nanowalls (VG) have recently been put forward as a potential candidate. Here, we report the use of VG covered by Si for increased supercapacitor performance. VG were grown on flexible graphite sheet (FGS) substrate by inductively coupled plasma chemical vapor deposition (ICP-CVD). Furthermore, silicon (Si) was deposited by magnetron sputtering on VG and the electrochemical performance studied in ionic liquid (IL) electrolyte. The incorporation of Si in VG/FGS provides an areal capacitance up to $16.4 \mathrm{mF} \mathrm{cm}^{-2}$, which is a factor 2 and 1.4 greater than that of bare substrate and VG/ FGS, respectively. This increase in capacitance does not penalize the cycling stability of Si/VG/GS, which remains outstanding up to 10,000 cycles in IL. In addition, the relaxation time constant decreases from 9.1 to 0.56 ms after Si deposition on VG/FGS.
\end{abstract}

\section{Introduction}

Nowadays the society demands for safe, flexible, reliable and lightweight energy storage devices like batteries and supercapacitors to run modern electronics [1-3]. Supercapacitors (SC) also named as electrochemical capacitors or electric double layer capacitors (EDLC) can deliver high energy densities comparable to those of batteries and high-power densities comparable to conventional capacitors [4]. Therefore, supercapacitor performance lies between conventional capacitors and batteries.

Supercapacitors rely on two mechanisms to store energy described as electrostatic charge storage and pseudocapacitance. The former consists in the formation of an electric double layer at the interface between electrode and electrolyte. Mainly large surface area carbon material and its composites are used as electrodes for EDLC. The latter consists in reversible and faradaic surface redox reactions taking place at the surface of conductive polymers, transitions metal oxides, or surface nitrogen-containing groups [5-7]. The performance of a SC depends on four important parameters: electrode material, electrolyte, current collector, and the electrode-current collector interface [8].

The use of a variety of nano-and/or composite materials as an electrode has made significant improvements in ion diffusion length and volumetric expansion issues during charge/discharge process [9-15]. Graphene, regarded as the most interesting material since Novoselov synthesized it in 2004 [16], is a two-dimensional structure made of a single layer of carbon atoms. Due to its remarkable properties, graphene has become a promising material for many applications like supercapacitors [17,18], Li-ion batteries [19,20] and solar cells [21].

The supercapacitor performance depends on the design and manufacturing process of the electrode materials [22-25]. In the case of

\footnotetext{
* Corresponding author. Department of Applied Physics, Universitat de Barcelona, C/Martí i Franquès, 1, 08028 Barcelona, Catalunya, Spain.

E-mail address: s.hussain@ulster.ac.uk (S. Hussain).
} 
SC, translation of graphene orientation from horizontal to vertical enhances $38 \%$ capacitance at scan rate of $100 \mathrm{mV} \mathrm{s}^{-1}$ due to high specific surface area as well as an open perpendicular structure that provides facile diffusion paths for ions [26]. The perpendicular structure of vertically aligned graphene nano-walls (GNWs) may also work as an open surface for deposition of other materials [27-29]. The specific capacitance delivered by carbon materials is lower than the one obtained as composites with certain metal oxides or polymers [30]. However, due to the faradic nature of their charge storage mechanism, these composites suffer from a lack of cycling stability and lower power density than the corresponding bare carbon material.

Silicon nanostructures are considered as a next generation electrode material for energy storage devices. It has been stated that $\mathrm{SiO}_{2}$ electrode materials display a very high differential capacitance $(180 \mu \mathrm{F}$ $\mathrm{cm}^{-2}$ ) in comparison to carbon electrodes (below $40 \mu \mathrm{F} \mathrm{cm}^{-2}$ ) [31]. Silicon and its derivatives such as $\mathrm{SiNW}$ ( $\mathrm{Si}$ nanowires), $\mathrm{SiO}_{2}, \mathrm{SiC}$ have been tested alone or in composite with other materials as EDLC by several researchers as presented in Table 1 [32-39].

Three types of electrolytes, namely aqueous, organic, and ionic liquids (IL) are mainly employed in supercapacitor manufacturing. The aqueous electrolytes are environmentally friendly, low-cost, non-flammable and have higher ionic conductivity. However, aqueous electrolytes have a small potential window of about $\sim 1.23 \mathrm{~V}$ [8]. On the other hand, organic electrolytes provide larger potential windows of $\sim 2.3 \mathrm{~V}$. However, they are toxic, flammable and expensive. Still, most commercial supercapacitors are constructed using organic electrolytes because they provide a larger potential window in comparison to aqueous electrolytes [40]. ILs are non-flammable, chemically and thermally stable and provide a potential window larger than $3 \mathrm{~V}$. Conversely, they are expensive and due to their high viscosity, supercapacitors display poor cycling stability and failure at high power [40]. Another significant factor influencing supercapacitor performance is the current collector. The prime contribution of the current collector in a supercapacitor is to let electrons flow efficiently between the active material and the external circuit during the working process. It is highly desirable to have a strong contact between the current collector and the active materials to minimize the series resistance of the device. Otherwise, a significant loss of energy would occur during the charging and discharging process [41].

Table 1

Capacitance comparison of Si and its derivates in different electrolytes.

\begin{tabular}{|c|c|c|c|}
\hline \multirow[t]{2}{*}{ Electrode } & \multirow[t]{2}{*}{ Electrolyte } & \multicolumn{2}{|l|}{ Capacitance } \\
\hline & & $\begin{array}{l}\text { Cyclic } \\
\text { Voltammetry }\end{array}$ & $\begin{array}{l}\text { Charge/ } \\
\text { Discharge }\end{array}$ \\
\hline $\begin{array}{l}\text { Mixture (MMPSiC: carbon } \\
\text { black: PVDF)/ } \\
\text { Aluminum foil [32] }\end{array}$ & $1 \mathrm{M} \mathrm{Na}_{2} \mathrm{SO}_{4}$ & $\begin{array}{l}253.7 \mathrm{Fg}^{-1} @ 5 \\
\mathrm{mV} \mathrm{s}^{-1}\end{array}$ & - \\
\hline $\begin{array}{l}\text { Mixture (MMPSiC: carbon } \\
\text { black: PVDF)/ } \\
\text { Aluminum foil [32] }\end{array}$ & $\begin{array}{l}\text { [EMIM] } \\
\text { [TFSI] }\end{array}$ & $\begin{array}{l}40.3 \mathrm{Fg}^{-1} @ 5 \\
\mathrm{mV} \mathrm{s}^{-1}\end{array}$ & - \\
\hline $\begin{array}{l}\text { SiNW on stainless steel } \\
\text { [33] }\end{array}$ & Et4NBF4 & $\begin{array}{l}\sim 58 \mathrm{~F} \mathrm{~g}^{-1} @ \sim \\
0.6 \mathrm{~A} \mathrm{~g}^{-1}\end{array}$ & - \\
\hline NiO coated SiNW [34] & $2 \mathrm{M} \mathrm{KOH}$ & - & $\begin{array}{l}787.500 \mathrm{~F} \mathrm{~g}^{-1} \\
@ 2.5 \mathrm{~mA}\end{array}$ \\
\hline $\begin{array}{l}\mathrm{SiO}_{2} \text { coated carbon cloth } \\
\text { [35] }\end{array}$ & $\begin{array}{l}\text { TEABF4/ } \\
\text { ACN }\end{array}$ & $\begin{array}{l}14.7 \mu \mathrm{F} \mathrm{cm} \mathrm{cm}^{-2} @ 2 \\
\mathrm{mV} \mathrm{s}^{-1}\end{array}$ & - \\
\hline SiNWs on $\mathrm{Si}[36]$ & $\begin{array}{l}1 \mathrm{M} \mathrm{NEt4BF4} \\
\text { in PC }\end{array}$ & $\begin{array}{l}46 \mu \mathrm{F} \mathrm{cm}{ }^{-2} @ 50 \\
\mathrm{mV} \mathrm{s}^{-1}\end{array}$ & - \\
\hline 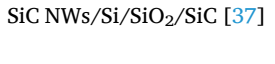 & $3.5 \mathrm{M} \mathrm{KCl}$ & $\begin{array}{l}400 \mu \mathrm{F} \mathrm{cm}^{-2} @ \\
100 \mathrm{mV} \mathrm{s}^{-1}\end{array}$ & - \\
\hline SiNWs/Si [38] & $1 \mathrm{M} \mathrm{KCl}$ & $\begin{array}{l}1.7 \mathrm{mF} \mathrm{cm} \mathrm{cm}^{-2} @ 50 \\
\mathrm{mVs}^{-1}\end{array}$ & - \\
\hline SiNWs/Si [39] & $1 \mathrm{M} \mathrm{Na}_{2} \mathrm{SO}_{4}$ & $\begin{array}{l}1.55 \mathrm{mF} \mathrm{cm}^{-2} \\
@ 10 \mathrm{mV} \mathrm{s}^{-1}\end{array}$ & - \\
\hline $\mathrm{MnO}_{2} / \mathrm{SiNWs} / \mathrm{Si}$ [39] & $1 \mathrm{M} \mathrm{Na}_{2} \mathrm{SO}_{4}$ & $\begin{array}{l}21.296 \mathrm{mF} \mathrm{cm}^{-2} \\
\text { @ } 10 \mathrm{mV} \mathrm{s}^{-1}\end{array}$ & - \\
\hline $\mathrm{Si} / \mathrm{VG} / \mathrm{FGs}$ [this work] & bmim- $\mathrm{MeSO}_{3}$ & $\begin{array}{l}16.4 \mathrm{mF} \mathrm{cm}^{-2} @ \\
10 \mathrm{mV} \mathrm{s}^{-1}\end{array}$ & $\begin{array}{l}8.4 \mathrm{mF} \mathrm{cm}{ }^{-2} \\
@ 0.4 \mathrm{~mA}\end{array}$ \\
\hline
\end{tabular}

In this work, vertically aligned graphene nanosheets (VG) were grown on flexible graphite sheet (FGS) substrate using inductively coupled plasma (ICP). Later, Silicon (Si) was sputtered on the surface of VG/FGS to prepare a nanocomposite for supercapacitor electrodes. Due to the instability of $\mathrm{Si}$ in aqueous electrolytes [36,38] an IL has been used to analyse its performance. The results show a good contact at the electrode/current collector interface, and an increase in cycling stability and power density after Si deposition. To the best of our knowledge, this is the first time that $\mathrm{Si} / \mathrm{VG}$ nanocomposites grown on flexible graphite current collector have been demonstrated for supercapacitor applications.

\section{Experimental}

Flexible graphite sheets (FGS) were purchased from Mersen Graphite company (Papyex flexible graphite sheet, n998) with a thickness of 0.02 $\mathrm{cm}$. Vertically oriented graphene (VG) nanostructures were grown directly on FGS by using inductively coupled plasma chemical vapor deposition (ICP-CVD) method. A detailed description of the ICP-CVD system has been reported previously [29]. FGS was introduced inside a quartz tubular reactor and the system was pumped down to a pressure below $1 \mathrm{~Pa}$. Afterwards, the synthesis of VG on FGS was performed as follows. The system was heated up to $750{ }^{\circ} \mathrm{C}$ without introducing any gas. After reaching the desired temperature, the precursor gas $\left(\mathrm{CH}_{4}, 10\right.$ $\mathrm{sccm}$ ) was introduced at a pressure of 50-60 $\mathrm{Pa}$ and the plasma ignited with an input power of $400 \mathrm{~W}$. The growth time of VG was set to $40 \mathrm{~min}$. Subsequently, the heating and plasma powers were switched off, as well as the $\mathrm{CH}_{4}$ flow. Finally, the system was let to cool down to room temperature. Pulsed DC magnetron sputtering was used for the sputtering process of $\mathrm{Si}(\sim 20 \mathrm{~nm}$ thick) on VG/FGS. The parameters used were: $120 \mathrm{~W}$ plasma power, $100 \mathrm{kHz}$ frequency of pulsed signal, $2016 \mathrm{~ns}$ pulse width, $20 \mathrm{sccm}$ of Ar gas flow and a working pressure of $1 \mathrm{~Pa}$. After the growth of the vertical nanostructures and in order to increase their surface energy, the VG were treated in situ at room temperature with a ICP of $40 \mathrm{~W}$ at a $\mathrm{O}_{2}$ pressure of $43 \mathrm{~Pa}$ for $30 \mathrm{~s}$. This post-treatment has a double effect of purifying the VG and increasing the wettability of the nanostructures, which facilitates contact with the electrolyte.

\section{Characterization techniques}

\subsection{Analysis methods}

The morphological analysis of the samples was performed using scanning electron microscopy (FESEM, HITACHI SU5000). The quality and structure of the samples were examined by micro-Raman spectroscopy (HORIVA LabRam HR800, Japan). A green laser of $532 \mathrm{~nm}$ wavelength, $0.5 \mathrm{~mW}$ power, and a 50LWD objective was used during the measurements. X-ray photoelectron spectroscopy (XPS) analysis was carried out using a Kratos AXIS ultra DLD with an Al K $\alpha(\mathrm{h} \nu=1486.6 \mathrm{eV})$ $\mathrm{X}$-ray source. The high-resolution spectra were deconvoluted using Shirley background subtraction and Gaussian-Lorentzian functions for the peak fitting (Casa XPS software). The electrochemical characterization of the samples was performed using a two-electrode cell configuration in non-aqueous electrolyte. The symmetrical supercapacitors were built in a MBRAUN Unilab dry glove box by sandwiching an ionic liquid (bmim- $\mathrm{MeSO}_{3}$ ) soaked separator (Whatman glassy-fiber GF/A) between two of the following electrodes: FGS, VG/ FGS and Si/VG/FGS. The geometrical area was $1.13 \mathrm{~cm}^{2}$ and the electrodes were analysed electrochemically using a potentiostat/galvanostat (Autolab PGSTAT30, Eco Chemie B.V., Utrecht, The Netherlands).

\subsection{Calculation formulas and methods}

The areal capacitance of one electrode calculated from cyclic voltammetry (CV) measurements was estimated using equations (1) and (2). 
$C=\left(q_{a}+\left|q_{c}\right|\right) /\left(2 \times \Delta U_{a p p l}\right)$

$C_{a}=2 \times C / A$

Where $C_{a}$ is the areal capacitance of a single electrode in $\mathrm{mF} \mathrm{cm}^{-2}, \mathrm{q}_{\mathrm{a}}$, and $\mathrm{q}_{\mathrm{c}}$ are the anodic and cathodic charges in $\mathrm{C}, C$ is the measured capacitance for the two-electrode cell and $A$ is the area of one electrode in $\mathrm{cm}^{2}[42,43]$.

The areal capacitance $\left(C_{a}\right)$ of a single electrode was calculated from charge-discharge curves of a complete device by the following equation (3).

$C_{a}=2 \times(I \times \Delta t) /(A \times \Delta U)$

Where $I$ is the discharge current in $\mathrm{mA}, \Delta t$ is the discharge time excluding IR drop due to the series resistance (ESR) in s, $\Delta U$ is the potential window in volt (V) excluding the voltage drop, $A$ is the area of one electrode material in $\mathrm{cm}^{2}$ [44].

Energy density and power density are two important parameters to evaluate the performance of SC for real applications. The energy density $\left(E_{a}\right)$ and volumetric power density $\left(P_{a}\right)$ were calculated using the following equations (4) and (5) [44].

$E_{a}=C(\Delta U)^{2} /(2 A \times 3600)$

$P_{a}=E_{a} \times 3600 / \Delta t$

Where $C$ is the capacitance calculated from the discharge curve in $\mathrm{F}, \Delta U$ is the voltage window and $\Delta t$ is the discharge time in $\mathrm{s}$ and $A$ the area of one electrode in $\mathrm{cm}^{2}$. The imaginary capacitance $\left(C^{\prime \prime}\right)$ is calculated using equation (6) [45].
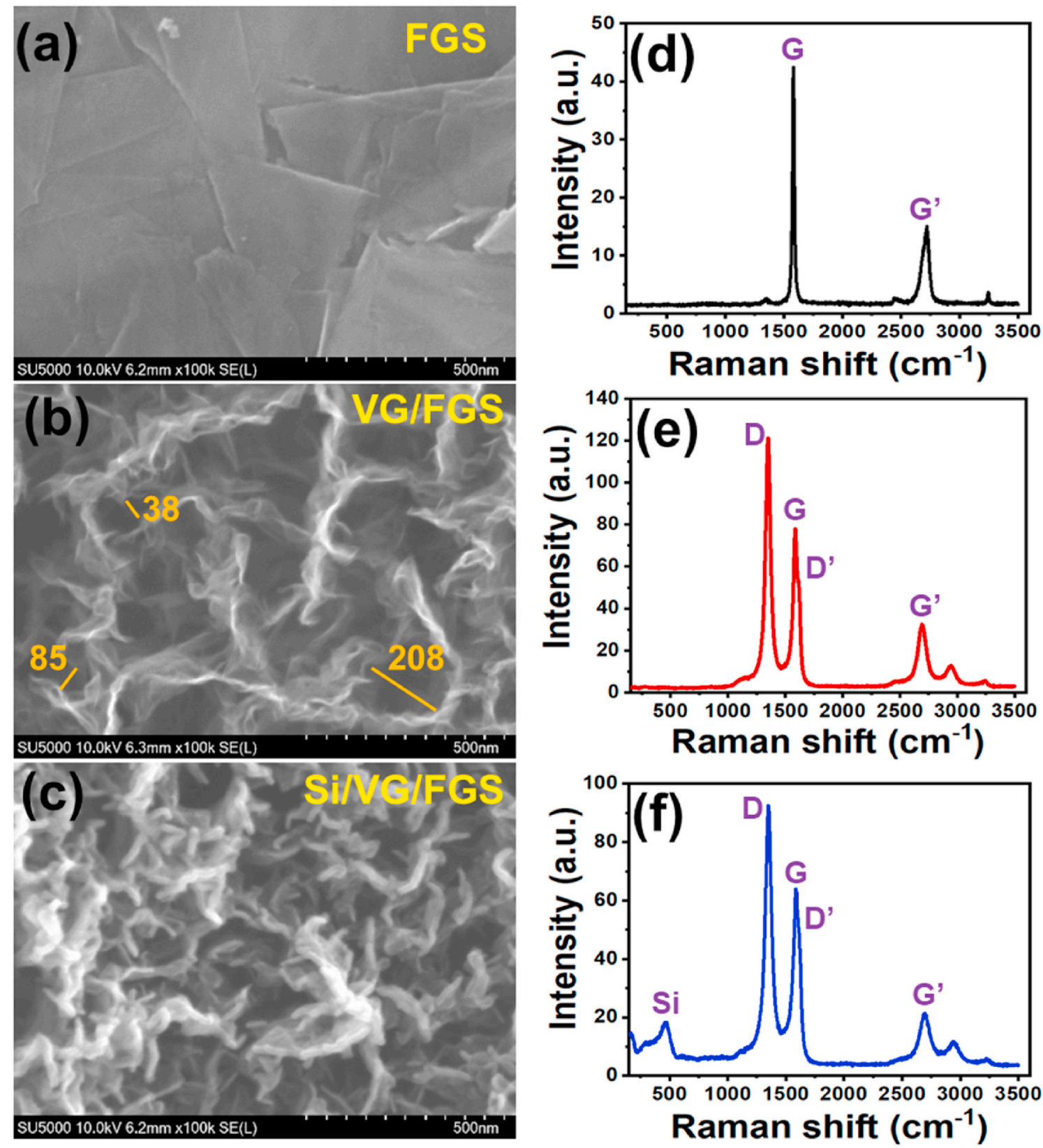

Fig. 1. SEM image of $\{(\mathrm{a})$ flexible graphite sheet (FGS), (b) Vertical graphene (VG) grown on FGS, lines and numbers indicate the pore size in nm, (c) Si deposited on VG\}. Raman spectra of $\{(\mathrm{d})$, flexible graphite sheet (FGS), (e), Vertical graphene (VG) grown on FGS, and (f), Si deposited on VG/FGS . 


$$
C^{\prime \prime}=\frac{-Z^{\prime}}{2 \pi f|Z|^{2}}
$$

Where $C^{\prime \prime}$ is the imaginary capacitance in $\mathrm{F}, Z^{\prime}$ is the real part of the impedance in $\Omega, f$ is the frequency in $\mathrm{Hz}$ and $|Z|$ is the modulus of the impedance in $\Omega$.

\section{Results and discussion}

\subsection{Microscopic and spectroscopic analysis}

Fig. 1 shows SEM images of FGS, VG/FGS and Si nanocluster decorated VG composite nanomaterial. The flexible graphite paper used as a substrate consists of parallel stacking of graphite sheets of different lengths visible in Fig. 1(a). The structure of VG grown on graphite papers is extremely porous, with big voids and wavy (Fig. 1(b)).

The VG are around $600 \mathrm{~nm}$ in length, a few nanometres thick and with sharp edges. The vertical nature of graphene with wider spaces between them makes it easier for carbon species to reach at the sides and bottom of the nanosheets. That is, it seems that geometry facilitate the creation of nucleation centres that lead to the formation of secondary nucleated VG structure. With the growth of secondary VG sheets, the density of the nanosheets or nanoflakes increases, which implies an enhancement in the surface area. This wider open structure (meso and macro pores) of VG is very convenient for further grafting of nanomaterials on the surface of graphene nanoflakes.

The wide voids provide good space to deposit other materials directly making good contact with graphene nanoflakes. Si deposited on VG has a maximum thickness of $\sim 20 \mathrm{~nm}$ (calibrated by sputtering $\mathrm{Si}$ on a flat surface) on sharp edges of nanoflakes and interlaced vertical graphene nanowalls. The sputtered Si does not disturb the wavy structure of the nanosheets. The $\mathrm{Si}$ atoms are located coating basal planes and edges of VG due to the non-directionality of the sputtering process. Nanosized clusters of $\mathrm{Si}$ are formed that can provide short diffusion pathways for ions and electrons, effectively diminishing the problem of pulverisation induced by large volumetric expansion [15].

Raman spectroscopy is a highly recommended technique to study the structural quality (disorder, defects, and doping level) of carbon-based nanomaterials. It also differentiates the structure of different carbon nanomaterials such as graphite, graphene, vertically oriented graphene, and carbon nanotubes. Raman spectroscopy displays bands based on the nature of the carbon nanomaterials at different wavenumbers.

The 1st and 2nd order Raman spectra of a carbon material displays most prominent peaks appearing at $\sim 1582 \mathrm{~cm}^{-1}$ (G band), which corresponds to $\mathrm{C}-\mathrm{C}$ stretching modes, $\mathrm{D}$ band at $\sim 1350 \mathrm{~cm}^{-1}$ and $\mathrm{D}^{\prime}$ band at $\sim 1620 \mathrm{~cm}^{-1}$, which are related to various kinds of lattice defects. A $2 \mathrm{D}\left(\mathrm{G}^{\prime}\right)$ band appears at $2700 \mathrm{~cm}^{-1}$, which is the 2 nd order of D mode [46,47]. Fig. 1(d) shows typical graphite Raman spectra of FGS with a very weak D peak, that can be attributed to a small number of structural defects. Raman spectra of VG/FGS exhibit four main peaks at (1350, 1587.2, 1618.2 and $2690.9 \mathrm{~cm}^{-1}$ ) corresponding to $\mathrm{D}, \mathrm{G}, \mathrm{D}^{\prime}$ and $\mathrm{G}^{\prime}$ bands respectively (Fig. 1(e)). $\mathrm{G}^{\prime}$ to $\mathrm{G}$ band intensity ratio provides an estimate of the number of layers [48]. The $I_{G}, I_{G}=0.42 \pm 0.04$ ratio confirms that the VG/FGS sample consists of a few layers of graphene nanoflakes. The quality of carbon materials is mainly identified by measuring the intensity ratio $I_{\mathrm{D}} / I_{\mathrm{G}}$ [49]. The value of this ratio is $1.69 \pm$ 0.07 and $1.60 \pm 0.06$ for VG/FGS and Si/VG/FGS, respectively. These similar values indicate that the $\mathrm{Si}$ deposition process does not damage the VG structure. The Raman spectra of SNC/VG/FGS presents an additional peak at $470 \mathrm{~cm}^{-1}$ [50], which confirms the presence of amorphous Si deposited on VG/FGS (Fig. 1(f)).

The elemental quantification was achieved from the XPS survey scan spectra (Fig. 2). The survey scan of FGS and VG/FGS demonstrates the presence of carbon and oxygen at $284.5 \mathrm{eV}$ and $531 \mathrm{eV}$ binding energies, respectively. $\mathrm{Si} / \mathrm{VG} / \mathrm{FGS}$ survey scan displays carbon and oxygen peaks

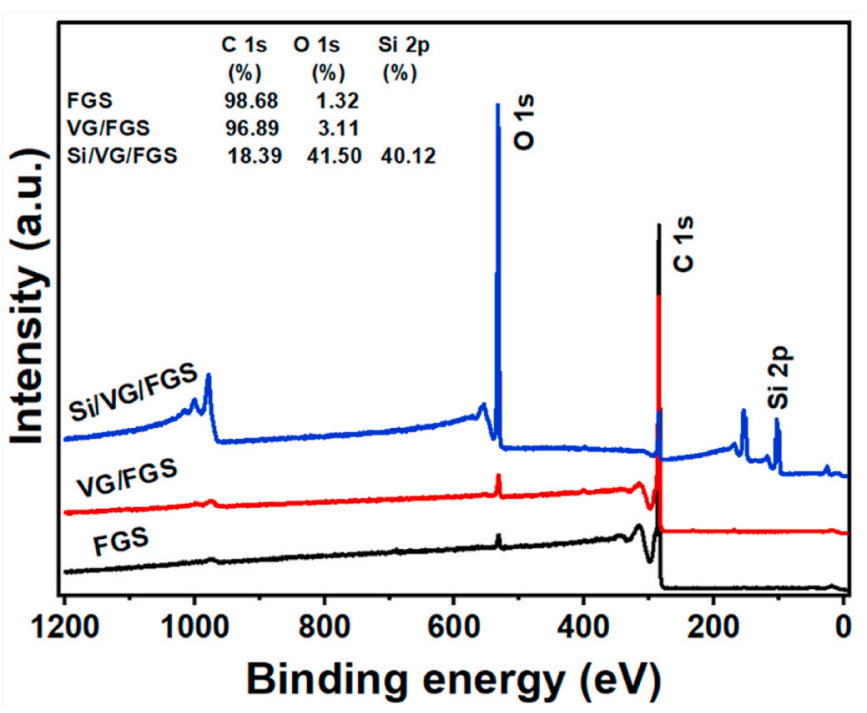

Fig. 2. XPS wide energy survey scan of flexible graphite sheet (FGS), Vertical graphene (VG) grown on FGS, and Si deposited on VG/FGS.

as well as a peak at $99 \mathrm{eV}$, which corresponds to $\mathrm{Si}$. The quantification analysis was performed at three different places of the sample to confirm its homogeneity. The elemental composition analysis reveals that the as received FGS sample consists of C1s (98.68 at.\%) and O1s (1.32 at.\%), VG/FGS sample consists of C1s (96.89 at.\%) and O1s (3.11 at.\%) and Si/ VG/FGS sample consists of C1s (18.39 at.\%), O1s (41.5 at.\%) and Si (40.12 at.\%). The higher ratio of oxygen in VG/FGS in comparison to FGS is probably due to the existence of defect sites and grain boundaries in the graphene, which readily react with atmospheric oxygen when the sample is removed from the reactor.

The high Si percentage and a small amount of carbon in the Si/VG/ FGS is in accordance with the Si coating on the surface of VG. The high oxygen percentage in the $\mathrm{Si} / \mathrm{VG} / \mathrm{FGS}$ sample is probably due to the existence of residual water molecules in the sputtering system and reaction of sputtered silicon with atmospheric moisture upon exposure to air.

The asymmetric shape of high resolution C1s spectra indicates the presence of other chemical moieties at the surface of the FGS, VG/FGS, and $\mathrm{Si} / \mathrm{VG} / \mathrm{FGS}$ as shown in Fig. 3. The deconvolution of C1s spectra for FGS and VG/FGS shows 9 peaks. The main C1s peak at $284.5 \pm 0.2 \mathrm{eV}$ was characterized as $\mathrm{C} 1(\mathrm{C}=\mathrm{C}) / \mathrm{sp}^{2}$ hybridized graphite like carbon. The $\mathrm{C} 2$ peak at $285.1 \pm 0.2 \mathrm{eV}$ was a feature of $\mathrm{C}-\mathrm{C} / \mathrm{sp}^{3}$ hybridized carbon atoms. The $\mathrm{C} 3$ peak at $285.8 \pm 0.1 \mathrm{eV}$ corresponds to the $\mathrm{C}-\mathrm{OH}$ chemical group. The $\mathrm{C} 4(\mathrm{C}-\mathrm{O})$ peak at $286.4 \pm 0.1 \mathrm{eV}, \mathrm{C} 5(\mathrm{C}=\mathrm{O})$ at $287.3 \pm 0.2$ $\mathrm{eV}$, and $\mathrm{C} 6(\mathrm{O}-\mathrm{C}=\mathrm{O})$ at $288.4 \pm 0.1 \mathrm{eV}$ indicate the presence of alcohol/ ether, carbonyl and carboxylic groups, respectively [11,51-53]. The C7 peak located at $291.0 \pm 0.1 \mathrm{eV}$ and $\mathrm{C} 8$ at $293.5 \pm 0.2 \mathrm{eV}$ were designated as shake up satellite (p-p*) and bulk-loss, respectively [54]. Due to the high number of defects in VG/FGS, the percentage of adsorbed impurities as oxygen functional groups is higher in comparison to FGS. In addition, a peak at $283.9 \pm 0.1 \mathrm{eV}$ was assigned to vacancy-like defects [53]. In the case of $\mathrm{Si} / \mathrm{VG} / \mathrm{FGS}, \mathrm{C} 1, \mathrm{C} 2$, and $\mathrm{C} 3$ peaks appear at the same binding energies as those for FGS and VG/FGS. For sample Si/VG/FGS the C4, C5, C6, C7, and C8 peaks were not detected, and the C9 peak is slightly shifted $\sim 0.5 \mathrm{eV}$ to lower binding energies. The disappearance of the oxygen functionalities and downshift of C9 is possibly due to changes in the local chemical environment of carbon related to the presence of $\mathrm{Si}$. The quantitative analysis of carbon attached to various oxygen groups for FGS, VG/FGS and $\mathrm{Si} / \mathrm{VG} / \mathrm{FGS}$ are provided in supplementary information (S1). Fig. 3(d) shows Si2p deconvoluted spectra of $\mathrm{Si} / \mathrm{VG} / \mathrm{FGS}$ in two elemental $\mathrm{Si}$ peaks, $\mathrm{S} 1\left(\mathrm{Si}_{2} \mathrm{p}_{3 / 2} ; 99.34 \pm 0.1 \mathrm{eV}\right)$ and $\mathrm{S} 2\left(\mathrm{Si} 2 \mathrm{p}_{1 / 2} ; 99.87 \pm 0.1 \mathrm{eV}\right)$, one complete oxide peak $\mathrm{S} 6\left(\mathrm{SiO}_{2}\right.$; $103.28 \pm 0.1 \mathrm{eV})$ and three sub-oxide peaks, $\mathrm{S} 3\left(\mathrm{Si}_{2} \mathrm{O} ; 100.09 \pm 0.1 \mathrm{eV}\right)$, 

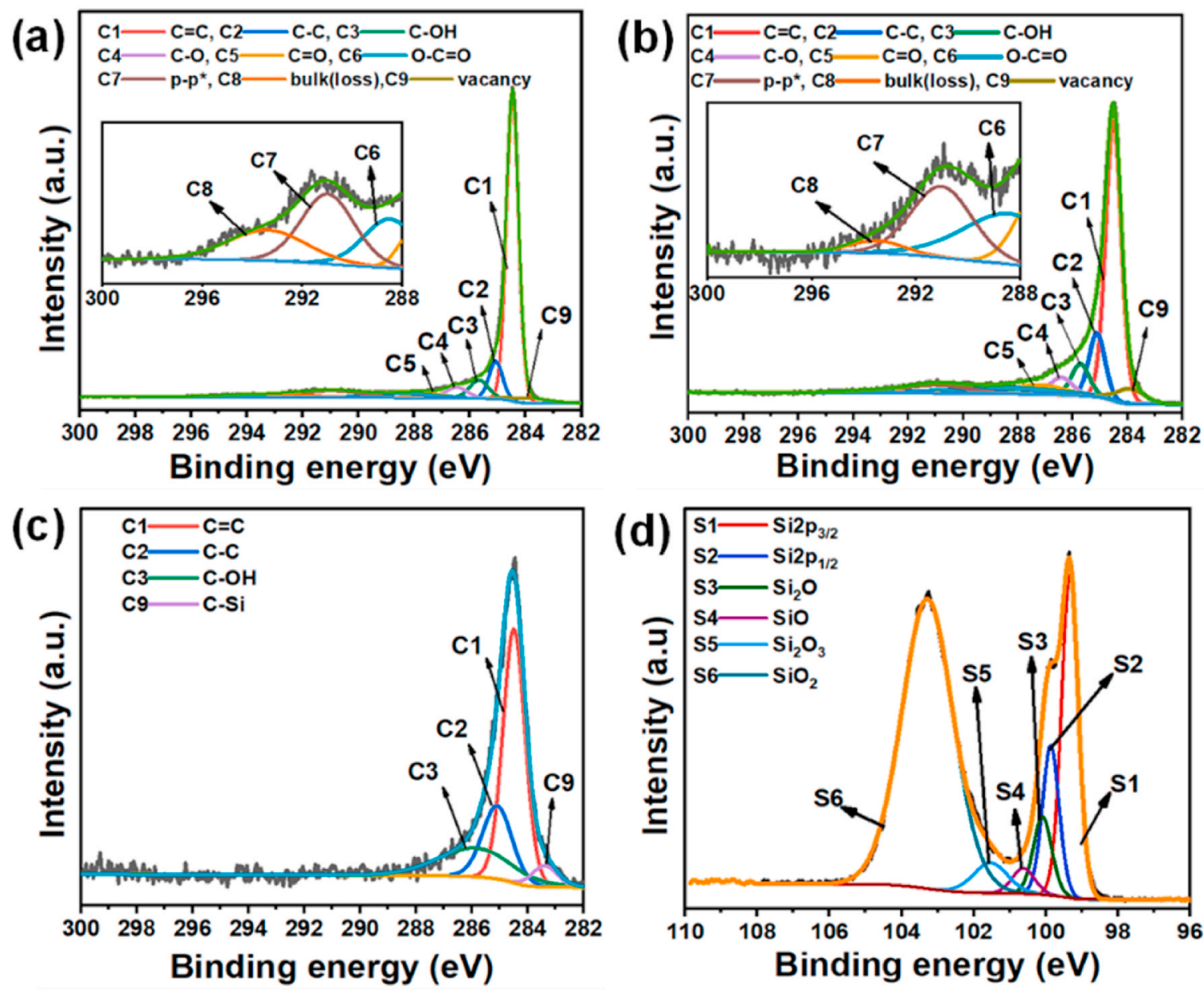

Fig. 3. High resolution deconvoluted spectra $\{\mathrm{C} 1 \mathrm{~s}$ (a) FGS, (b) VG/FGS, (c) Si/VG/FGS $\},\{\mathrm{Si} 2 \mathrm{p}$ (d) $\mathrm{Si} / \mathrm{VG} / \mathrm{FGS}$.

S4 $(\mathrm{SiO} ; 100.63 \pm 0.1 \mathrm{eV})$ and $\mathrm{S} 5\left(\mathrm{Si}_{2} \mathrm{O}_{3} ; 101.54 \pm 0.1 \mathrm{eV}\right)[55,56]$. Supplementary information (S2) shows that the concentration of elemental silicon is 30.58 at. $\%$ and the rest (69.42 at.\%) consists of $\mathrm{SiO}_{2}$ and sub-oxides.

\subsection{Electrochemical measurements}

Supercapacitors tested in IL at different scan rates $\left(5-150 \mathrm{mV} \mathrm{s}^{-1}\right)$ in the voltage range of $0-1.8 \mathrm{~V}$, show typical rectangular-shape voltammograms (Fig. 4(a)). As anticipated, the CV of Si/VG/FGS presents a larger integrated area and current in comparison to other samples. The CV curves display two different non-faradic and faradic capacitance regimes at different potentials. The charge storage mechanism presented by VG/FGS is mainly electrostatic (electric double layer capacitance) as can be seen from the linear increase in voltage until $1.65 \mathrm{~V}$, above this voltage current increases exponentially which can be related to faradaic charge transfer due to minor oxygen functional groups at the surface of graphene. Samples FGS and Si/VG/FGS start to have significant faradaic charge transfer at $1 \mathrm{~V}$ or below. The faradaic charge transfer taking place at the electrode-electrolyte interface for FGS is due to moisture (oxygen groups) at the surface of graphite sheets. For $\mathrm{Si} / \mathrm{VG} / \mathrm{FGS}$ the emergence of faradaic charge transfer was mainly due to the presence of $\mathrm{Si}$ oxide derivatives. Fig. 4(b) shows a comparison of the capacitance at different scan rates. The delivered capacitances for (FGS, VG/FGS, and Si/VG/
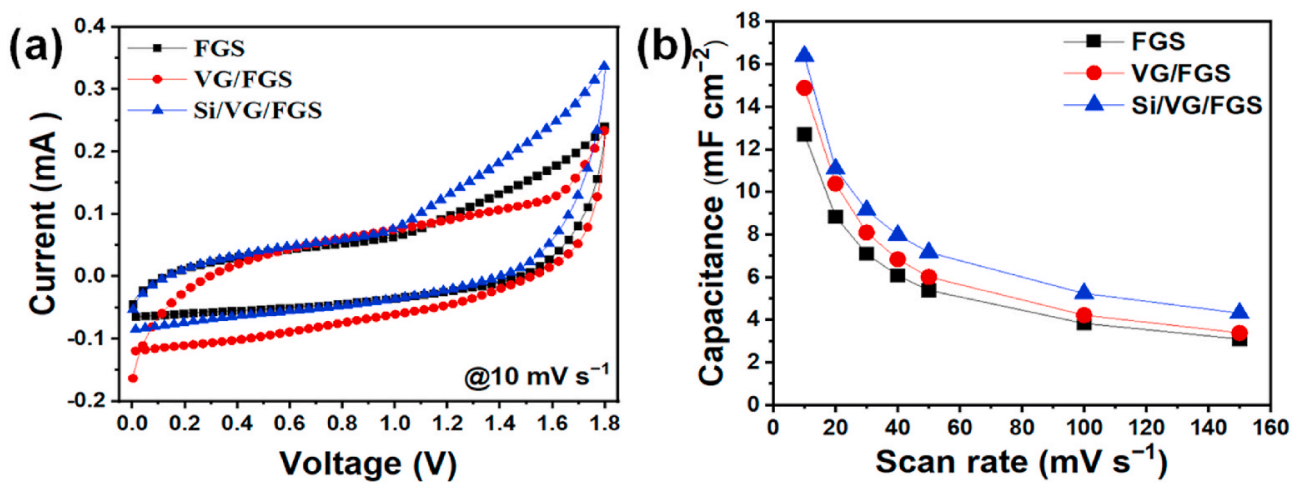

Fig. 4. (a) Cyclic voltammetry comparison between samples at $10 \mathrm{mV} \mathrm{s}^{-1}$ scan rate and (b) capacitance comparison at different scan rates. 
FGS) are $\left(12.7,14.9\right.$ and $\left.16.4 \mathrm{mF} \mathrm{cm}^{-2}\right)$ respectively, at scan rate $10 \mathrm{mV}$ $\mathrm{s}^{-1}$. The highest capacitance obtained for $\mathrm{Si} / \mathrm{VG} / \mathrm{FGS}$ is related to the well-anchored Si to VG. The capacitance decreases as the scan rate increases because at lower scan rates electrolyte ions have more time to diffuse into almost the whole available space of the electrode material. At high scan rates $\left(150 \mathrm{mV} \mathrm{s}^{-1}\right)$ sample ( $\left.\mathrm{Si} / \mathrm{VG} / \mathrm{FGS}\right)$ delivers the highest capacitance of $4.3 \mathrm{mF} \mathrm{cm} \mathrm{cm}^{-2}$.

Fig. 5(a) presents galvanostatic charge-discharge measurements of FGS, VG/FGS and Si/VG/FGS at a constant current of $0.4 \mathrm{~mA}$ in the potential window $0-1.8 \mathrm{~V}$. The charge-discharge profiles display a symmetrical shape for all samples. The capacitance of a single electrode from two electrode cell configuration was calculated using equation (5). The capacitance values of (FGS, VG/FGS, and $\mathrm{Si} / \mathrm{VG} / \mathrm{FGS}$ ) were (3.6, 6.1, 8.4 and $\mathrm{mF} \mathrm{cm}^{-2}$ ) respectively (see Fig. 5(b)). The decrease in capacitance with an increase in current could be related to ohmic resistance along the path of micropores and/or to ion diffusion constraints through the porous structure surface [57].

The long-term charge-discharge stability of the supercapacitors was evaluated by charging and discharging at $0.5 \mathrm{~mA}$ current during 10,000 cycles (Fig. 5(c)). SNC/VG/FGS sample shows an increase in capacitance from (6.5-7.1 $\mathrm{mF} \mathrm{cm}^{-2}$ ) after 3500 cycles most probably due to improvement in electrolyte wetting with time. In addition, due to a large pore structure and electrostatic charge storage mechanism, this sample presents a stable capacitance during 10,000 cycles. VG/FGS sample shows a decrease in capacitance from $3.8 \mathrm{mF} \mathrm{cm}^{-2}$ to $3.5 \mathrm{mF} \mathrm{cm}^{-2}$ in the first few cycles. Afterwards, it keeps an almost constant capacitance value of $3 \mathrm{mF} \mathrm{cm}^{-2}$ up to 10,000 cycles, which corresponds to $79 \%$ capacitance retention. In contrast, $\mathrm{Si} / \mathrm{VG} / \mathrm{FGS}$ sample shows a $106 \%$ capacitance retention after the same number of charge/discharge cycles, which demonstrates its suitability as a nanocomposite for supercapacitor applications.

Energy density and power density are two important parameters to evaluate the performance of supercapacitors for real applications. The energy density $(E)$ and power density $(P)$ were calculated using equations (6) and (7). The corresponding Ragone plot of the samples is shown in Fig. 5(d), where it can be seen that Si/VG/FGS delivers the highest

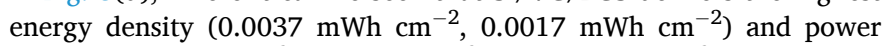
density $\left(0.7 \mathrm{~mW} \mathrm{~cm}{ }^{-2}, 1.8 \mathrm{~mW} \mathrm{~cm}^{-2}\right)$ at $0.35 \mathrm{~mA} \mathrm{~cm}^{-2}$ and $0.88 \mathrm{~mA}$ $\mathrm{cm}^{-2}$ currents densities respectively, in comparison to other samples.

The supercapacitors were further analysed by electrochemical impedance spectroscopy (EIS). The results are presented using the Nyquist plot in Fig. 6, where the inset shows the high frequency region. The ESR values for (FGS, VG/FGS, and Si/VG/FGS) were (3.5, 3.6 and $2.9 \Omega \mathrm{cm}^{2}$ ) respectively. The low ESR values demonstrate excellent contact between $\mathrm{Si}, \mathrm{VG}$, and graphite sheet $[58,59]$, which also leads to enhanced ionic conductivity through the inner structure of the electrode. There was no appearance of semicircle for all samples, which indicates that double layer capacitance is the main charge storage mechanism. At low frequencies, the trend of the slope $\mathrm{Si} /$ VG/FGS $>$ VG/FGS $>$ FGS manifests that amorphous $\mathrm{Si}$ nanoclusters are well-anchored to VG/FGS and promote ionic diffusion into Si pores, graphene, and graphite sheet. Thus, direct growth of VG results in strong adhesion to the flexible graphite sheet substrate, which diminishes contact resistance. In addition, its vertical structure allows fast ion diffusion and, consequently, a decrease in the ESR.

The imaginary capacitance $\left(C^{\prime \prime}\right)$ was calculated by equation 8 . The $C^{\prime \prime}$ vs. frequency plot shows a maximum at the relaxation frequency $f_{0}$ that normally appears at $45^{\circ}$ phase angle in phase versus frequency plots. The frequency $f_{0}$ separate the resistive and capacitive parts of the supercapacitor. The inverse of $f_{0}$ is called the relaxation time constant $\left(\tau_{0}\right)$, which can be calculated by the following equation $\left(\tau_{0}=1 / f_{0}\right)$ as shown in Fig. 6(b). Supercapacitors require low values of $\tau_{0}$ in order to deliver high power densities. The relaxation time constant for VG/FGS is $9.1 \mathrm{~ms}$ and $0.56 \mathrm{~ms}$ for $\mathrm{Si} / \mathrm{VG} / \mathrm{FGS}$. These results indicate that VG composite with amorphous $\mathrm{Si}$ nanocluster promote fast ion
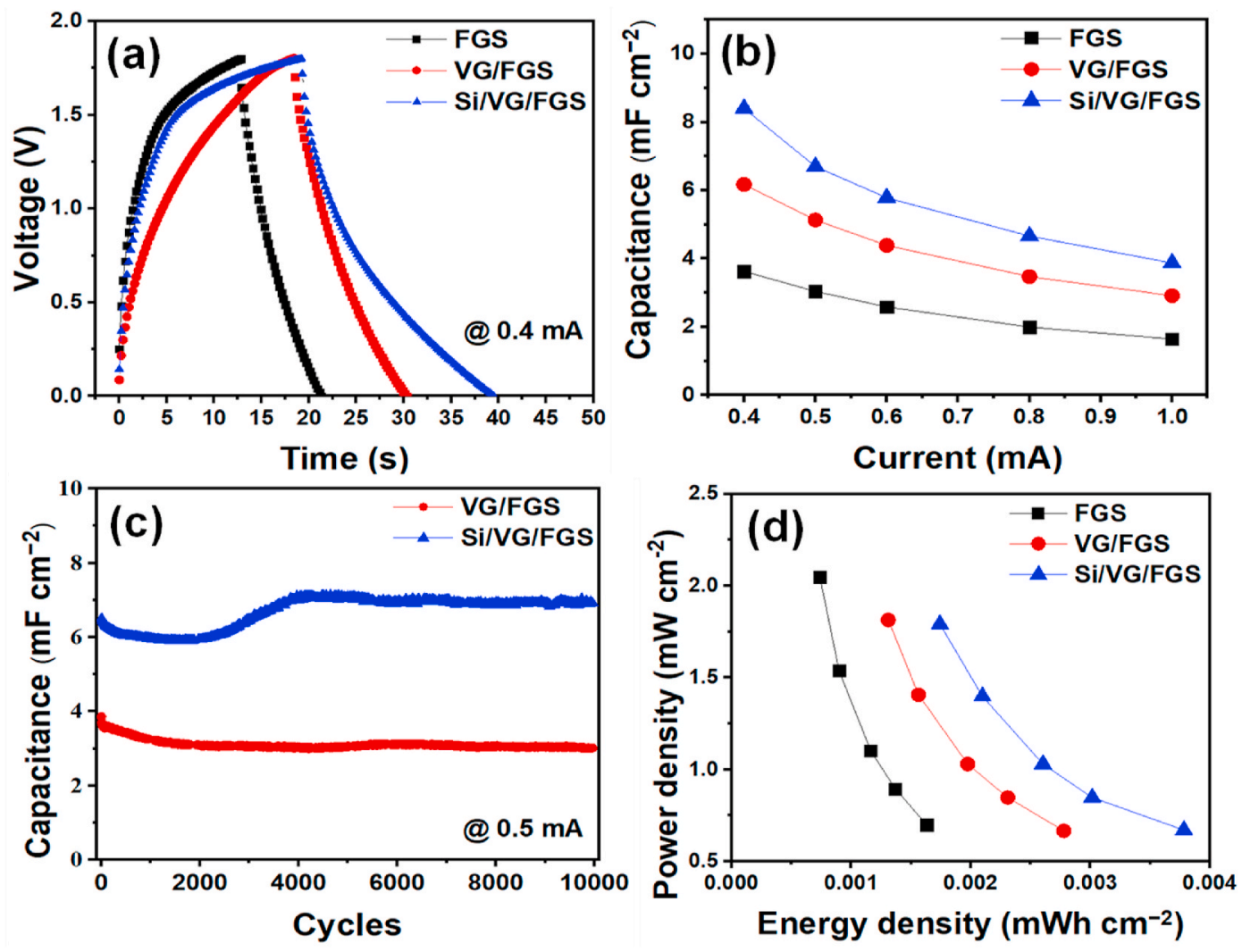

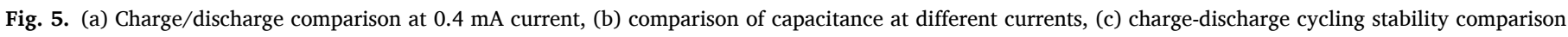
and (d) Ragone plot comparison at different current densities. 

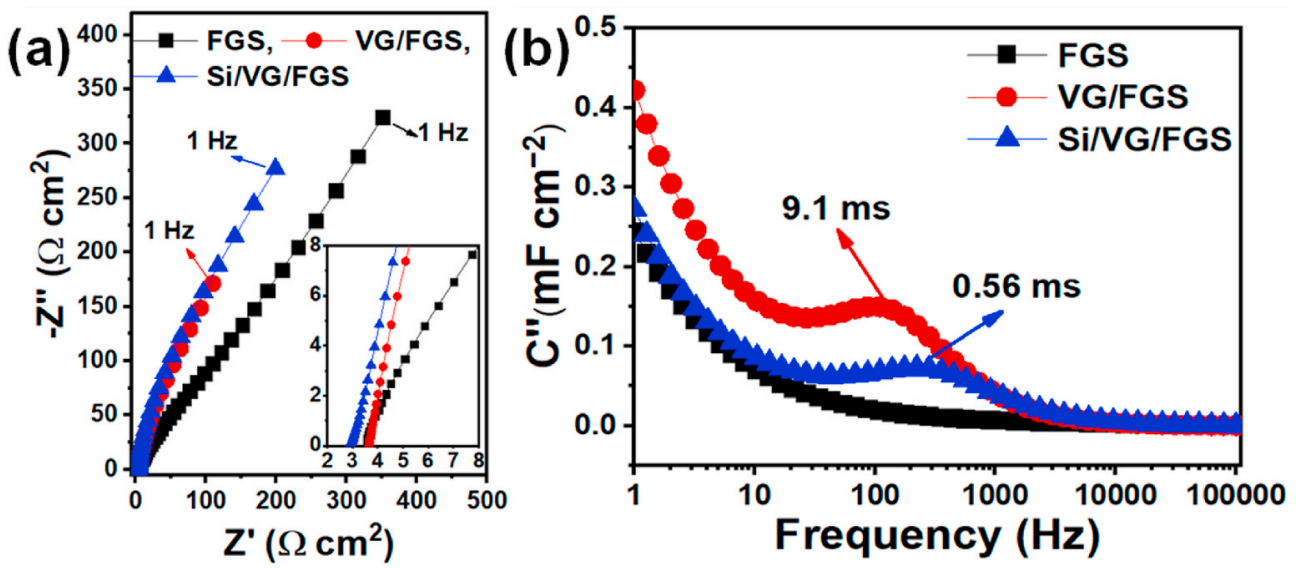

Fig. 6. (a) Nyquist plot for FGS, VG/FGS and Si/VG/FGS, (b) Variation of imaginary capacitance with frequency.

transportation through the pores of the composite electrode material, which results in a better power delivery nature [60]. The sudden increase in $\mathrm{C}^{\prime \prime}$ at very low frequencies is due to the contribution of faradic capacitance [61]. We did not observe the peak maximum for FGS sample in $C^{\prime \prime}$ vs frequency plot. The absence of this peak could be due to leakage current or a wide distribution of porous structure $[62,63]$.

\section{Conclusion}

We have manufactured $\mathrm{Si} / \mathrm{VG} / \mathrm{FGS}$ nanocomposite electrodes by vapor deposition methods. Morphological and spectroscopic results reveal the formation of few layer vertical graphene nanoflakes grown on flexible graphite sheet. The deposited silicon nanoclusters were determined to be amorphous in nature and cover the surface of vertical graphene from top to bottom. The Si/VG/FGS composite show a 2 and 1.4-fold increase in capacitance with respect to FGS and VG/FGS respectively, a high cyclic reversibility up to 10,000 cycles at $0.5 \mathrm{~mA}$ and a very small relaxation time constant of $0.56 \mathrm{~ms}$ in IL. The energy and power densities were for $\mathrm{Si} / \mathrm{VG} / \mathrm{FGS}$ than those of FG and VG/FGS. These results show the potential of $\mathrm{Si} /$ graphene nanowalls composite electrode as high power and energy density electrodes in IL.

\section{Declaration of competing interest}

The authors declare that they have no known competing financial interests or personal relationships that could have appeared to influence the work reported in this paper.

\section{Acknowledgements}

The authors acknowledge financial support of the Spanish Ministry of Economy, Industry and Competitiveness (MINECO) under Project No. ENE2017-89210-C2-2-R. The ENPHOCAMAT (FEMAN) group acknowledges support from the AGAUR of Generalitat de Catalunya, Project No. 2017SGR0985. Two authors (A.M.-A. and J.M.-G.) acknowledge the financial support from their respective APIF grants from Universitat de Barcelona. The authors thank the CCiT-UB technicians for their support in the characterization techniques used to obtain the results of this publication.

\section{Appendix A. Supplementary data}

Supplementary data to this article can be found online at https://doi. org/10.1016/j.ceramint.2021.04.190.

\section{References}

[1] Maria R. Lukatskaya, Bruce Dunn, Yury Gogotsi, Multidimensional materials and device architectures for future hybrid energy storage, Nat. Commun. 7 (2016) 12647.

[2] Chuanyin Xiong, Mengrui Li, Wei Zhao, Chao Duan, Lei Dai, Mengxia Shen, Yongjian Xu, Yonghao Ni, A smart paper@polyaniline nanofibers incorporated vitrimer bifunctional device with reshaping, shape-memory and self-healing properties applied in high-performance supercapacitors and sensors, Chem. Eng. J. 396 (2020) 125318.

[3] Chi Xia, Yibing Xie, Hongxiu Du, Wei Wang, Ternary nanocomposite of polyaniline/manganese dioxide/titanium nitride nanowire array for supercapacitor electrode, J. Nanoparticle Res. 17 (2015) 30.

[4] So Yeun Kim, Bo-Hye Kim, Silica decorated on porous activated carbon nanofiber composites for high-performance supercapacitors, J. Power Sources 328 (2016) 219-227.

[5] Faxing Wang, Xiongwei Wu, Xinhai Yuan, Zaichun Liu, Yi Zhang, a Lijun Fu, Yusong Zhu, Qingming Zhou, Yuping Wu, Wei Huang, Latest advances in supercapacitors: from new electrode materials to novel device designs, Chem. Soc. Rev. 46 (2017) 6816.

[6] Shahzad Hussain, Roger Amade, Eric Jover, Enric Bertran, Nitrogen plasma functionalization of carbon nanotubes for supercapacitor applications, J. Mater. Sci. 48 (2013) 7620-7628.

[7] Chuanyin Xiong, Mengrui Li, Shuangxi Nie, Weihua Dang, Wei Zhao, Lei Dai, Yonghao Ni, Non-carbonized porous lignin-free wood as an effective scaffold to fabricate lignin-free Wood@Polyaniline supercapacitor material for renewable energy storage application, J. Power Sources 471 (2020) 228448.

[8] Nicklas Blomquist, Thomas Wells, Britta Andres, Joakim Bäckström, Sven Forsberg, Hakan Olin, Metal-free supercapacitor with aqueous electrolyte and low-cost carbon materials, Sci. Rep. 7 (2017) 39836.

[9] Chuanyin Xiong, Weihua Dang, Shuangxi Nie, Chengrong Qin, Dongping Li, Lei Dai, Mengxia Shen, Yongjian Xu, Yonghao Ni, Fabrication of high value cellulose nanofibers@Ni foam by non carbonization: various application developed during the preparation, Cellulose 28 (2021) 1455-1468.

[10] Daraghmeh Allan, Shahzad Hussain, Atta Ul Haq, Iyad Saadeddin, Llorenç Servera, J.M. Rui, Carbon nanocomposite electrodes for electrical double layer capacitor, J. Energy Storage 32 (2020) 101798.

[11] Shahzad Hussain, Eva Kovacevic, Roger Amade, Johannes Berndt, Cedric Pattyn, Ana Dias, Chantal Boulmer-Leborgne, Mohamed-Ramzi Ammar, Enric BertranSerra, Plasma synthesis of polyaniline enrobed carbon nanotubes for electrochemical applications, Electrochim. Acta 268 (2018) 218-225.

[12] Ting-Feng Yi, Ying Li, Yan-Mei Li, Shaohua Luo, Yan-GuoLiu, ZnS nanoparticles as the electrode materials for high-performance supercapacitors, Solid State Ionics 343 (2019) 115074

[13] Chongjun Zhao, Yaoxuan Huang, Chunhua Zhao, Xiaoxiao Shao, Zhaoqiang Zhu, Rose-derived 3D carbon nanosheets for high cyclability and extended voltage supercapacitors, Electrochim. Acta 291 (2018) 287-296.

[14] Ramesh J. Deokate, Ramchandra S. Kalubarme, Chan-Jin Park, Chandrakant D. Lokhande, Simple synthesis of $\mathrm{NiCo}_{2} 4$ thin films using spray pyrolysis for electrochemical supercapacitor application: a Novel approach, Electrochim. Acta 224 (2017) 378-385.

[15] Guanhua Lin, Hongchun Wang, Ling Zhang, Qijin Cheng, Zhengliang Gong, Kostya (Ken) Ostriko, Graphene nanowalls conformally coated with amorphous/ nanocrystalline Si as high-performance binder-free nanocomposite anode for lithium-ion batteries, J. Power Sources 437 (2019) 226909.

[16] F. Schedin, A.K. Geim, S.V. Morozov, E.W. Hill, P. Blake, M.I. Katsnelson, K. S. Novoselov, Detection of individual gas molecules adsorbed on graphene, Nat. Mater. 6 (2007) 652-655.

[17] Debosmita Banerjee, Kamatchi Jothiramalingam Sankaran, Sujit Deshmukh, Mateusz Ficek, Gourav Bhattacharya, Jacek Ryl, Deodatta Maheshwar Phase, Mukul Gupta, Robert Bogdanowicz, I-Nan Lin, Aloke Kanjilal, Ken Haenen, Susanta 
Sinha Roy, 3D hierarchical boron-doped diamond-multilayered graphene nanowalls as an efficient supercapacitor electrode, J. Phys. Chem. C 123 (2019) 15458-15466.

[18] Yu Zhou, LeLe Wen, Ke Zhan, Ya Yan, Bin Zhao, Three-dimensional porous graphene/nickel cobalt mixed oxide composites for high-performance hybrid supercapacitor, Ceram. Int. 44 (2018), 17 21848-21854.

[19] Yuhai Hu, Xifei Li, Dongsheng Geng, Mei Cai, Ruying Li, Xueliang Sun, Influence of paper thickness on the electrochemical performances of graphene papers as an anode for lithium ion batteries, Electrochim. Acta 91 (2013) 227-233.

[20] Wen Wang, Mingqi Tang, Zhenwei Yan, Superior Li-storage property of an advanced LiFePO4@C/S-doped graphene for lithium-ion batteries, Ceram. Int. 46 (2020), 14 22999-23005.

[21] A.M. Afzal, Yasir Javeed, Sajad Hussain, Adnan Ali, M.Z. Yaqoob, Sohail Mumtaz, Enhancement in photovoltaic properties of bismuth ferrite/zinc oxide heterostructure solar cell device with graphene/indium tin oxide hybrid electrodes, Ceram. Int. 46 (2020), 7 9161-9169.

[22] Chuanyin Xiong, Bingbing Li, Chao Duan, Lei Dai, Shuangxi Nie, Chengrong Qin, Yongjian Xu, Yonghao Ni, Carbonized wood cell chamber-reduced graphene oxide@PVA flexible conductive material for supercapacitor, strain sensing and moisture-electric generation applications, Chem. Eng. J. 418 (2021) 129518.

[23] Xiuxia Zuo, Xiaoyan Wang, Yonggao Xia, Shanshan Yin, Qing Ji, Zhaohui Yang, Meimei Wang, Xiaofang Zheng, Bao Qiu, Zhaoping Liu, Jin Zhu, Peter Muller Buschbaum, Ya-Jun Cheng, Silicon/carbon lithium-ion battery anode with 3D hierarchical macro-/mesoporous silicon network: self-templating synthesis via magnesiothermic reduction of silica/carbon composite, J. Power Sources 412 (2019) 93-104.

[24] Hui Wu, Gerentt Chan, Wook Jang, Choi, Ill Ryu, Yan Yao, Matthew T. McDowell, Seok Woo Lee, Ariel Jackson, Yang Yuan, Liangbing Hu, Yi Cui, Stable cycling of double-walled silicon nanotube battery anodes through solid-electrolyte interphase control, Nat. Nanotechnol. 7 (2012) 310-315.

[25] Chuanyin Xiong, Bingbing Li, Heguang Liu, Wei Zhao, Chao Duan, Haiwei Wu, Yonghao Ni, A smart porous wood-supported flower-like NiS/Ni conjunction with vitrimer co-effect as a multifunctional material with reshaping, shapememory, and self-healing properties for applications in high-performance supercapacitors, catalysts, and sensors, J. Mater. Chem. A 8 (2020) 10898.

[26] Yu Zhang, Qionghui Zou, Hua Shao Hsu, Supil Raina, Yuxi Xu, Joyce B. Kang, Jun Chen, Shaozhi Deng, Ningsheng Xu, Weng P. Kang, Morphology effect of vertical graphene on the high performance of supercapacitor electrode, ACS Appl. Mater. Interfaces 8 (2016) 7363-7369.

[27] Eftekhari Ali, The mechanism of ultrafast supercapacitors, J. Mater. Chem. A 6 (2018) 2866.

[28] Ty Mai Dinh, Achour Amine, Sorin Vizireanu, Gheorghe Dinescu, Leona Nistor, Kevin Armstrong, Daniel Guay, David Pech, Hydrous $\mathrm{RuO}_{2}$ /carbon nanowalls hierarchical structures for all-solid-state ultrahigh-energy-density microsupercapacitors, Nano Energy 10 (2014) 288-294.

[29] Roger Amade, Arevik Muyshegyan-Avetisyan, Joan Marti Gonzalez, Angel Perez del Pino, Eniko Gyorgy, Esther Pascual, Jose Luis Andujar, Enric Bertran Serra, Super-capacitive Performance of manganese dioxide/graphene nano-walls electrodes deposited on stainless steel current collectors, Materials 12 (2019) 483

[30] Lei Qian, Lu Lu, Fabrication of three-dimensional porous graphene-manganese dioxide composites as electrode materials for supercapacitors, Colloid. Surface. Physicochem. Eng. Aspect. 465 (2015) 32-38.

[31] Norsaadatul Akmal, Mohd Zaid, Nurul Hayati Idris, Enhanced capacitance of hybrid layered graphene/nickel nanocomposite for supercapacitors, Sci. Rep. 6 (2016) 32082.

[32] Myeongin Kim, Ilgeun Oh, Jooheon Kim, Superior electric double layer capacitor using micro- and mesoporous silicon carbide sphere, J. Mater. Chem. A 3 (2015) 3944.

[33] Wook Jang, Choi, James McDonough, Sangmoo Jeong, Jee Soo Yoo, Candace K. Chan, Yi Cui, Stepwise nanopore evolution in one-dimensional nanostructures, Nano Lett. 10 (2010) 1409-1413.

[34] Fang Lu, Mengchun Qiu, Xiang Qi, Liwen Yang, Jinjie Yin, Hao Guolin, Feng Xiang, Jun Li, Jianxin Zhong, Electrochemical properties of high-power supercapacitors using ordered NiO coated Si nanowire array electrodes, Appl. Phys. A 104 (2011) $545-550$.

[35] C. Kevin, Wendy E. Leonarda, Marc A. Anderson Suyama, Improvement of electrochemical capacitor electrodes using $\mathrm{SiO}_{2}$ nanoparticles, Electrochim. Acta 56 (2011) 10137-10144.

[36] Fleur Thissandier, Annaïg Le Comte, Olivier Crosnier, Gentile Pascal, Gérard Bidan, Emmanuel Hadji, Thierry Brousse, Saïd Sadki, Highly doped silicon nanowires based electrodes for micro-electrochemical capacitor applications, Electrochem. Commun. 25 (2012) 109-111.

[37] Maxime Vincent, Mun Sek Kim, Carlo Carraro, Roya Maboudian, Silicon carbide Nanowires as an Electrode Material for High Temperature Supercapacitor, MEMS 2012, https://doi.org/10.1109/MEMSYS.2012.6170088. Paris, FRANCE, 29 January - 2 February 2012

[38] John P. Alper, Maxime Vincent, Carlo Carraro, Roya Maboudian, Silicon carbide coated silicon nanowires as robust electrode material for aqueous microsupercapacitor, Appl. Phys. Lett. 100 (2012) 163901.

[39] Fatsah Moulai, Toufik Hadjersi, Madjid Ifires, Adel Khen, Nacera Rachedi, Enhancement of electrochemical capacitance of silicon nanowires arrays (SiNWs) by modification with manganese dioxide $\mathrm{MnO}_{2}$, Silicon 11 (2019) 2799-2810.

[40] Zhong Cheng, Yida Deng, Wenbin Hu, Jinli Qiao, Zhangd Lei, Jiujun Zhang, A review of electrolyte materials and compositions for electrochemica supercapacitors, Chem. Soc. Rev. 44 (2015) 7484.
[41] Bo Zheng, Weiguang Zhu, Wei Ma, Zhenhai Wen, Xiaorui Shuai, Junhong Chen, Jianhua Yan, Zhihua Wang, Kefa Cen, Xinliang Feng, Vertically oriented graphene bridging active-layer/current-collector interface for ultrahigh rate supercapacitors, Adv. Mater. 25 (2013) 5799-5806.

[42] Shahzad Hussain, Roger Amade, Enric Bertran, Study of CNTs structural evolution during water assisted growth and transfer methodology for electrochemical applications, Mater. Chem. Phys. 148 (2014) 914-922.

[43] S. Jiang, T. Shi, X. Zhan, H. Long, S. Xi, H. Hu, Z. Tang, High-performance all-solidstate flexible supercapacitors based on two-step activated carbon cloth, J. Power Sources 272 (2014) 16-23.

[44] Meryl D. Stoller, Rodney S. Ruoff, Best practice methods for determining an electrode material's performance for ultracapacitors, Energy Environ. Sci. 3 (2010) 1294-1301.

[45] V. Ganesh, S. Pitchumanib, V. Lakshminarayanan, New symmetric and asymmetric supercapacitors based on high surface area porous nickel and activated carbon, J. Power Sources 158 (2006) 1523-1532.

[46] M.R. Ammar, E. Charon, J.-N. Rouzaud, J. Aleon, G. Guimbretière, P. Simon, On a reliable structural characterization of polished carbons in meteorites by Raman Microspectroscopy, Spectrosc. Lett. 44 (2011) 535-538.

[47] A. Pimenta, G. Dresselhaus, M.S. Dresselhaus, L.G. Cancado, A. Jorio, R. Saito, Studying disorder in graphite-based systems by Raman spectroscopy, Phys. Chem. Chem. Phys. 9 (2007) 1276-1290.

[48] Duhee Yoon, Hyerim Moon, Hyeonsik Cheong, Jin Sik Choi, Jung Ae Choi, Bae Ho Park, Variations in the Raman spectrum as a function of the number of graphene layers, J. Kor. Phys. Soc. 55 (2009) 1299-1303.

[49] Shahzad Hussain, Eva Kovacevic, Johannes Berndt, Neelakandan, M. Santhosh, Cédric Pattyn, Ana Dias, Thomas Strunskus, Mohamed-Ramzi Ammar, Andrea Jagodar, Mireille Gaillard, Chantal Boulmer-Leborgne, Uroš Cvelbar, Lowtemperature low-power PECVD synthesis of vertically aligned graphene, Nanotechnology 31 (2020) 395604.

[50] Won-Eui Hong, Jae-Sang Ro, Kinetics of solid phase crystallization of amorphous silicon analyzed by Raman spectroscopy, J. Appl. Phys. 114 (2013), 073511.

[51] P.L. Girard-Lauriault, J.C. Ruiz, T. Gross, M.R. Wertheimer, W.E.S. Unger, Ultrashallow chemical characterization of organic thin films deposited by plasma and vacuum-ultraviolet, using angle- and excitation energy-resolved XPS, Plasma Chem. Plasma Process. 31 (2011) 535-550.

[52] Imre Bertoti, Miklos Mohai, Krisztina Laszlo, Surface modification of graphene and graphite by nitrogen plasma: determination of chemical state alterations and assignments by quantitative X-ray photoelectron spectroscopy, Carbon 84 (2015) $185-196$.

[53] K. Ganesan, Subrata Ghosh, Nanda Gopala Krishna, S. Ilango, M. Kamruddin, A. K. Tyagi, A comparative study on defect estimation using XPS and Raman spectroscopy in few layer nanographitic structures, Phys. Chem. Chem. Phys. 18 (2016) 22160.

[54] Pierre-Luc Girard-Lauriault, René Illgen, Juan-Carlos Ruiz, Michael R. Wertheimer, Wolfgang E.S. Unger, Surface functionalization of graphite and carbon nanotubes by vacuum-ultraviolet photochemical reactions, Appl. Surf. Sci. 258 (2012) 8448-8454.

[55] December 19th 2012 Muhammad Y. Bashouti, Matthias Pietsch, Kasra Sardashti, Gerald Brönstrup, Sebastian W. Schmitt, Sanjay K. Srivastava, Jürgen Ristein, Jordi Arbiol, Hossam Haick, Silke Christiansen, Hybrid silicon nanowires: from basic research to applied nanotechnology, nanowires - recent advances, Xihong Peng, IntechOpen, 10.5772/54383. Available from: https://www.intechopen.co $\mathrm{m}$ /books/nanowires-recent-advances/hybrid-silicon-nanowires-from-basic-resear ch-to-applied-nanotechnology.

[56] Wa Li Zhang, Sam Zhang, Ming Yang, Tu Pei Chen, Microstructure of magnetron sputtered amorphous SiOx films: formation of amorphous Si core-Shell nanoclusters, J. Phys. Chem. C 114 (2010) 2414-2420.

[57] Itir Bakis Dogru, Mete Batuhan Durukan, Onur Turel, Husnu Emrah Unalan, Flexible supercapacitor electrodes with vertically aligned carbon nanotubes grown on aluminum foils, Prog. Nat. Sci.: Materials International 26 (2016) 232-236.

[58] Xianzhong Sun, Xiong Zhang, Haitao Zhang, Dacheng Zhang, Yanwei Ma, A comparative study of activated carbon-based symmetric supercapacitors in $\mathrm{Li}_{2} \mathrm{SO}_{4}$ and $\mathrm{KOH}$ aqueous electrolytes, J. Solid State Electrochem. 16 (2012) $2597-2603$.

[59] Vanchiappan Aravindan, Joe Gnanaraj, Yun-Sung Lee, Srinivasan Madhavi, Insertion-type electrodes for nonaqueous Li-ion capacitors, Chem. Rev. 114 (2014) 11619-11635.

[60] Bidhan Pandit, Babasaheb R. Sankapal, Highly conductive energy efficient electroless anchored silver nanoparticles on MWCNTs as a supercapacitive electrode, NewJ.Chem. 41 (2017) 10808.

[61] Simonetta Palmas, Michele Mascia, Annalisa Vacca, Javier Llanos, Esperanza Mena, Analysis of photocurrent and capacitance of $\mathrm{TiO}_{2}$ nanotube-polyaniline hybrid composites synthesized through electroreduction of an aryldiazonium salt, RSC Adv. 4 (2014) 23957.

[62] Dipendu Saha, Yunchao Li, Zhonghe Bi, Jihua Chen, Jong, K. Keum, Dale K. Hensley, Hippolyte A. Grappe, Harry M. Meyer, Sheng Dai, M. Parans Paranthaman, A.K. Naskar, Studies on supercapacitor electrode material from activated lignin-derived mesoporous carbon, Langmuir 30 (2014) 900-910.

[63] Jong H. Jang, Songhun Yoon, Bok H. Ka, Yong-Ho Jung, Seung M. Oh, Complex capacitance analysis on leakage current appearing in electric double-layer capacitor carbon electrode, J. Electrochem. Soc. 152 (2005) A1418-A1422. 